\title{
Eric Verdeil, Thomas Ansart, Benoit Martin, Patrice Mitrano, Antoine Rio, Atlas des mondes urbains
}

Clément Dillenseger

Doctorant à l'École urbaine de Lyon et à I'ENS de Lyon.

$18 / 01 / 2021$

Selon le mode de calcul choisi, entre cinq et sept humains sur dix vivraient en ville. C'est en partant de ce constat statistique que le géographe Eric Verdeil et les cartographes Thomas Ansart, Benoît Martin, Patrice Mitrano et Antoine Rio ont élaboré l'Atlas des Mondes Urbains. Ce deuxième opus d'une collection des Presses de Science Po lancée en 2019 reprend le modèle de son prédécesseur, l'Atlas de l'Anthropocène, et repose sur un dialogue réussi entre le texte et l'image. L'objectif de cet ouvrage, énoncé dès l'introduction, est de corriger les défauts un « imagier trompeur » des mondes urbains qui serait dominant dans les représentations et qui mettrait trop en avant le gigantisme des «métropoles XXL ». Loin de correspondre à cet imaginaire, les réalités urbaines sont en fait plurielles : il existe des formes et des tailles de villes variées ainsi que des manières différentes de vivre, de gouverner et de fabriquer les mondes urbains.

En plus de présenter le projet du livre, l'introduction comprend une partie fort intéressante sur la manière de mesurer et de définir les villes et le taux d'urbanisation. Il s'agit d'un travail de présentation des outils statistiques et des sources disponibles qui atteste d'une rigueur intellectuelle appréciable. On apprend ainsi que, si chaque État définit différemment les villes, un outil élaboré par la commission européenne (le Global Human Settlement Layer) permet de créer une base de donnée universelle fondée sur l'utilisation d'images satellites.

La suite de l'ouvrage est composée de sept chapitres différents que l'on a choisi d'associer ainsi : les chapitres «Accélération » et « Morphologies » proposent des éléments de contexte et forment une première partie. Les chapitres «Métropolisation», «Vies Urbaines » et «Anthropocène » constituent une deuxième partie : ils fournissent des grilles 
de lectures de l'état de l'urbain contemporain et de son fonctionnement. Enfin, les chapitres «Politiques » et «Expériences » permettent, dans une troisième partie, de rappeler que les mondes urbains ne sont pas spontanés mais résultent d'arbitrages sociaux et politiques permanents.

Contextuelle, la première partie permet donc de restituer la variété du fait urbain et des formes urbaines dans le temps et dans l'espace. C'est l'occasion pour les auteurs de revenir sur des processus et des concepts tels que la transition urbaine (p. 24-25) ou l'étalement et la verticalisation (p. 36-37). Le lecteur a également le plaisir de découvrir les multiples façons dont les villes grandissent, que ce soit d'un point de vue historique (p.2021), démographique (p. 26-27) ou spatial (p. 38-39). Cette première partie donne également à voir une qualité transversale à l'ensemble de l'ouvrage: grâce à une grande attention portée aux échelles d'analyse, le discours est toujours nuancé et rigoureux. Cela est par exemple illustré par la double-page sur les périphéries des villes qui s'appuie sur le cas d'Istanbul (p. 44-45). En complément du texte, des dessins font advenir les quartiers périphériques mentionnés qu'une carte permet de situer.

La seconde partie rend encore plus concrète cette présentation de l'urbain en s'intéressant à la manière dont les villes fonctionnent, économiquement et écologiquement, mais aussi en plaçant la focale sur les habitants et la manière dont ils vivent la ville au quotidien. Les thématiques choisies permettent de rappeler des réalités parfois mises de côté par l'imagier traditionnel de la ville. Par exemple, le fait que les villes reposent, aujourd'hui encore, beaucoup sur les industries (p. 58-59) et pas uniquement sur les activités tertiaires le plus souvent vantées. D'autres données permettent de tordre le coup à certaines idées reçues. Ainsi, les villes illustrent l'échec de la «théorie du ruissellement » qui justifie pourtant la course contemporaine à "l'attractivité »: cent villes détiennent à elles seules $46 \%$ des richesses urbaines... qui ne bénéficient qu'à $25 \%$ de la population (p. 50-51). Ces données ne sont pas que des chiffres mais correspondent à la ville telle qu'elle est vécue par les populations les plus vulnérables : le milieu urbain est un espace où les inégalités sont particulièrement accrues, comme l'illustrent les cas de São Paulo (p. 70-71) et de New- 
York (p. 90-91). Ces inégalités socio-spatiales sont redoublées par les changements environnementaux qui touchent l'ensemble des villes dans le monde (p. 88-89)... alors même que l'empreinte environnementale de ces villes est une des causes majeures des bouleversements écologiques (p. 82-83).

Face à ces défis sociaux et écologiques, comment s'orienter ? La troisième partie aborde la gouvernance des mondes urbains à différents échelons. Elle mentionne tour à tour le rôle des États, des municipalités, des réseaux de ville, des entreprises et des habitants dans la gestion et la planification des territoires urbains. La ville est l'objet de nombreuses rivalités mais elle est aussi le support et le lieu d'expression de nombreuses luttes. Une tonalité critique, présente dès le début de l'Atlas, est particulièrement saillante dans les deux derniers chapitres. Ce positionnement est bienvenu car il souligne que les gagnants des politiques urbaines ne sont pas toujours les habitants comme le montrent, entre autres, l'exemple de la création des «villes nouvelles» (p. 96-97) ou celui de la gestion de la ressource en eau (p. 102-103). Pour autant, les habitants des villes disposent parfois d'un pouvoir qui leur permet d'être des acteurs politiques de premier rang : en élisant des majorités sensibles aux enjeux environnementaux, comme à Fribourg-en-Brisgau (Allemagne) (p. 122-123) ou en bricolant des solutions aux problèmes du quotidien, comme c'est le cas à Accra (Ghana), où les autorités tendent à reconnaître l'efficacité des transports informels (p. 130-131).

En définitive, l'Atlas des Mondes Urbains est un ouvrage extrêmement riche. Superbement réalisé d'un point de vue graphique, très bien écrit et astucieusement construit, il offre un large panorama de situations urbaines contemporaines. Les exemples sont puisés sur tous les continents et concernent des villes de tailles variées : l'objectif de décentrement énoncé en introduction est ainsi atteint. L'environnement et 
les inégalités sont abordés de manière transversale ${ }^{1}$ et rendent le propos éminemment actuel. Loin d'être lisse, l'ouvrage rappelle ainsi que la politique et la ville sont intrinsèquement (étymologiquement!) liées. Très pédagogique, le propos ne nie pas pour autant la complexité des mondes urbains. Il est ainsi à conseiller aux étudiants en sciences sociales dès la licence mais il peut également trouver une audience "grand public ». S'il faut souligner la beauté et la variété des figures, certaines (rares !) sont parfois difficiles à s'approprier, voire simplement à lire. Ne voyons là que le prix à payer d'un véritable parti pris pour l'innovation graphique qui reste une qualité plus qu'un défaut.

${ }^{1}$ Jusque dans la conception matérielle du livre, imprimé en France et répondant à des normes durables de production. 